\title{
Curcumin Inhibits Proliferation of Epstein-Barr Virus-Associated Human Nasopharyngeal Carcinoma Cells by Inhibiting EBV Nuclear Antigen 1 Expression
}

\author{
Limei Liu $\left(\mathbb{D},{ }^{1}\right.$ Jiaomin Yang $\mathbb{D}^{2},{ }^{2}$ Wuguang $J i\left(\mathbb{D},{ }^{3}\right.$ and Chao Wang $\mathbb{D i D}^{4}$ \\ ${ }^{1}$ Department of Keratonosus, Weifang Eye Hospital, Weifang 261053, Shandong, China \\ ${ }^{2}$ Department of Laboratory Medicine, WeifangYidu Central Hospital, Qingzhou 262500, Shandong, China \\ ${ }^{3}$ Department of Vascular Surgery, Weifang Peoples's Hospital, Weifang 261053, Shandong, China \\ ${ }^{4}$ Department of Pathogen Biology, Weifang Medical University, Weifang 261053, Shandong, China
}

Correspondence should be addressed to Chao Wang; wangchao96988@126.com

Received 6 May 2019; Revised 31 August 2019; Accepted 12 September 2019; Published 7 October 2019

Academic Editor: Himanshu Garg

Copyright (c) 2019 Limei Liu et al. This is an open access article distributed under the Creative Commons Attribution License, which permits unrestricted use, distribution, and reproduction in any medium, provided the original work is properly cited.

\begin{abstract}
This investigation aims to study the effect of curcumin on the proliferation, cycle arrest, and apoptosis of Epstein-Barr virus(EBV-) positive nasopharyngeal carcinoma (NPC) cells. $\mathrm{EBV}^{+} \mathrm{NPC}$ cells were subjected to curcumin treatment. The cell viability was evaluated with the CCK-8. Cell cycle and apoptosis were analyzed by flow cytometry analysis. Expression (protein and mRNA) levels were detected with western blotting and quantitative real-time PCR, respectively. Curcumin efficiently reduced the viability of $\mathrm{EBV}^{+}$NPC cells. Curcumin induced the cycle arrest of the HONE1 and HK1-EBV cells positive for EBV. Moreover, curcumin treatment promoted the NPC cell apoptosis, via the mitochondria- and death receptor-mediated pathways. Furthermore, curcumin decreased the expression of EBNA1 in the HONE1 and HK1-EBV cells and inhibited the transcriptional level of EBNA1 in the HeLa cells. Curcumin induced EBNA1 degradation via the proteasome-ubiquitin pathway. In addition, curcumin inhibited the proliferation of HONE1 and HK1-EBV cells positive for EBV, probably by decreasing the expression level of EBNA1. In both the HONE1 and HK1-EBV cells, curcumin inhibited the EBV latent and lytic replication. Curcumin could reduce the EBNA1 expression and exert antitumor effects against NPC in vitro.
\end{abstract}

\section{Introduction}

Epstein-Barr virus (EBV), a well-known common $\gamma$-herpes virus, induces asymptomatic infection in approximately $95 \%$ adults throughout the world [1]. The EBV infection has been regarded as the high-risk factor related to more than $1 \%$ cancer cases [2]. Moreover, the infection of EBV has also been implied in various proliferative diseases associated with epithelial or lymphoid cells in human beings, such as the gastric carcinoma, nasopharyngeal carcinoma (NPC), Burkitt's lymphoma, and Hodgkin's lymphoma [3]. As a malignant tumor, NPC mainly originates from the nasopharynx epithelium, which is endemic in South China and Southeast Asia, and the disease incidence is $20-50$ cases per 100,000 people $[4,5]$. In NPC, EBV infection has type II latency mechanism [6], which is featured by several noncoding RNAs, latent membrane proteins (LMP1, LMP2A, and LMP2B), and Epstein-Barr nuclear antigen 1 (EBNA1) [7]. Due to the special viral antigen expression, NPC has become an attractive target for clinical therapies.

The essential viral protein EBNA1 is expressed in tumors associated with EBV, during the viral latency processes (but not for latency 0) [8]. EBNA1 plays vital roles in the maintenance, replication, and segregation of latent episomes [9]. Moreover, EBNA1 could interact with the cellular and viral promoters, regulating the gene transcription level [9]. A recent study has shown that EBNA1 has oncogenic activity, and this pleiotropic protein regulates its own synthesis to escape from the host cells exerting immune functions [10]. Furthermore, EBNA1 has also been shown to be able to 
decrease p53 accumulation in the epithelial cells infected with EBV, which might disrupt the antitumor function of these cells [11]. Although expressed in malignancies associated with EBV, the EBNA1 expression would obey various regulating modes, which depends on the types of host cells. Under most circumstances, the EBNA1 transcription would be promoted by the Q promoter (Qp) (in both type I or II latency) [12]. For the NPC cells (type II infection), Qp could mediate EBNA1 transcription [13]. Furthermore, it has been suggested that EBNA1 would be necessary the tumor cell proliferation [14]. EBNA1 is recognized as an attractive target in various therapeutic strategies in clinics.

Curcumin (Cur) is a polyphenol extracted from Curcuma longa, which has been extensively investigated due to its modulating effects of signaling pathways related to proliferation and invasion of cells, as well as angiogenesis $[15,16]$. Curcumin can exert pharmacological functions, such as antimicrobial and anti-inflammation functions [17-19]. Moreover, curcumin is able to block the cancer growth of various types, including prostate cancer, NPC, and other cancers [20,21]. Curcumin could reduce human breast cancer cell (MDA-MB-231) proliferation by decreasing the expression level of Bcl-2 and upregulating the Bax, PARP, and cleaved caspase 3 expression levels [22]. A previous study has shown that curcumin could sensitize the NPC cells to radiation via modulating the noncoding RNA levels, Jab1/CSN5, and/ or ROS generation [23]. Furthermore, curcumin is shown to cause NPC cell apoptosis through both the mitochondria-dependent and death receptor-mediated pathways [24].

Our previous study had shown that heat shock protein 90 (Hsp90) inhibitors could block the proliferation of malignant cells infected with EBV, both in vivo and in vitro [25]. It has also been suggested that triptolide treatment could inhibit $\mathrm{EBV}^{+} \mathrm{B}$ lymphocyte proliferation through inhibiting LMP1 [26]. Furthermore, we have also shown that berberine shows the antitumor activity against NPC through the EBNA 1-dependent mechanism [13]. Furthermore, triptolide could decrease the human telomerase reverse transcriptase stability in BC-3 and BCBL-1 cells [27].

In this study, effects of curcumin on cell proliferation, cellular apoptosis, and cycle arrest of $\mathrm{EBV}^{+}$ NPC cells were investigated. Moreover, mechanisms through which curcumin inhibited NPC were also analyzed.

\section{Materials and Methods}

2.1. Study Cells. $\mathrm{EBV}^{+}$NPC cells (i.e., the HONE1 and HK1EBV cells) were kind gifts from Prof. SaiWahTsao (University of Hong Kong, Hong Kong, China). These cells were cultured with the RPMI-1640 medium, which contained G418 (400 ng/ml) and 10\% fetal bovine serum (Gibco-BRL). HeLa cells were cultured in the DMEM medium, which also contained $10 \%$ fetal bovine serum. These cells were cultured at $37^{\circ} \mathrm{C}$ with $5 \% \mathrm{CO}_{2}$.
2.2. Cellular Viability Assessment. Cellular viability was analyzed with the Cell Counting Kit-8 (CCK-8) (Dojindo Laboratories). The cells were planted onto the 96-well plate $\left(1 \times 10^{4}\right.$ cells $\left./ 100 \mu \mathrm{l}\right)$, which were incubated with DMEM. These cells were incubated with $0.006 \%$ DMSO (vehicle control) or increasing concentrations of curcumin (SigmaAldrich, Shanghai, China), for 24 and $48 \mathrm{~h}$, respectively. Then, $10 \mu \mathrm{l}$ CCK- 8 was added to incubate the cells in dark at $37^{\circ} \mathrm{C}$ for $1 \mathrm{~h}$. The $\mathrm{OD}_{450}$ value was detected with an $\mathrm{EL} \times 800$ microimmuno analyzer (BioTek Instruments, Inc.).

2.3. Flow Cytometry. Annexin V-FITC/PI apoptosis detection kit (Multisciences) was used to analyze apoptosis. HK1-EBV and HONE1 cells were incubated with $0.006 \%$ DMSO (vehicle control) or curcumin $(5 \mu \mathrm{M}$ and $10 \mu \mathrm{M})$. After $24 \mathrm{~h}$, cells were resuspended in the binding buffer $(500 \mu \mathrm{l})$. The cells were incubated with Annexin V-FITC $(5 \mu \mathrm{l})$ and PI $(10 \mu \mathrm{l})$. After that, cells were analyzed with EPICS Altra II (Beckman Coulter).

2.4. Transfection. The plasmids of pSG-5-EBNA1 and pGL3.0-Qp were synthesized by the Neuron Biotech Corporation (Shanghai, China). Transient cellular transfection was performed with X-tremeGENE HP DNA Transfection Reagent (Roche). After $4 \mathrm{~h}$ of transfection, 0.006\% DMSO (control) or curcumin was added and incubated for $44 \mathrm{~h}$.

2.5. Dual Luciferase Reporter Assay. After cotransfection with $100 \mathrm{ng}$ pGL3.0-Qp and $5 \mathrm{ng}$ pRL-TK for $4 \mathrm{~h}$, HeLa cells were incubated with $0.006 \%$ DMSO or curcumin (5 or $10 \mu \mathrm{M})$. After $24 \mathrm{~h}$, the total protein was harvested with the $1 \mathrm{X}$ lysis buffer (Promega, Madison, WI, USA). Luciferase activity was measured by the GLO-MAX 20/20 system (Promega, Madison, WI, USA). Renilla was as internal control reference.

2.6. Quantitative Real-Time PCR. The total RNA was extracted with TRIzol (Invitrogen, Grand Island, NY, USA). First-strand cDNA was synthesized with the Reverse Transcription kit (Takara, Tokyo, Japan). Quantitative realtime PCR was conducted on the CFX96 real-time PCR detection system, with the SYBR Premix Ex Taq kit (Takara, Tokyo, Japan). Primer sequences were as follows: EBNA1, forward $5^{\prime}$-GGTCGTGGACGTGGAGAAAA-3' and reverse $5^{\prime}$-GGTGGAGACCCGGATGATG-3'; and GAPDH, forward $5^{\prime}$-ACATCGCTCAGACACCATG-3' and reverse 5'-TGTAGTTGAGGTCAATGAAGGG-3'. The reaction conditions were set as follows: $95^{\circ} \mathrm{C}$ for $30 \mathrm{~s} ; 95^{\circ} \mathrm{C}$ for $10 \mathrm{~s}$, $62^{\circ} \mathrm{C}$ for $10 \mathrm{~s}$, and $72^{\circ} \mathrm{C}$ for $15 \mathrm{~s}$, for a total of 45 cycles. The house-keeping gene GAPDH was as internal reference.

2.7. Western Blotting. Cells were lysed with the RIPA buffer (Beyotime, Shanghai, China), containing 0.5\% cocktail protease inhibitor (Roche) and $0.5 \mathrm{mM}$ PMS, on a microscraper. After sonication for $15 \mathrm{~s}$, the extract was centrifuged $(12,000 \times g)$ for $15 \mathrm{~min}$. Protein concentration was 
determined with the BCA method (standard sample: bovine serum albumin). Proteins were separated with $10 \%$ SDSPAGE and transferred onto the PVDF membrane. After blocking with $5 \%$ skim milk in TBST for $1 \mathrm{~h}$, the PVDF membrane was cultured with the anti-caspase-3 (Cat. \#9668; CST, CA, USA), anti-mouse anti-EBNA1 (Cat. \#sc81581; Santa Cruz, Santa Cruz, CA, USA), anti-caspase-9 (Cat. \#A26361; ABclonal, Boston, UK), anti-cleaved caspase-3 (Cat. \#9664; CST), anti-p53 (Cat. \#sc-126; Santa Cruz), anticleaved PARP-1 (Cat. No. sc-56, 196; Santa Cruz), antiGAPDH (Cat. \#10494-1-AP; Proteintech, Wuhan, Hubei, China), anti-Fas (Cat. \#8023; CST), and anti-FasL (Cat. \#4273; CST), respectively, at $4^{\circ} \mathrm{C}$ overnight. Then, the membrane was washed with TBST and then cultured with the HRP-conjugated anti-rabbit IgG (Wuhan Keri Technology) and anti-mouse IgG (Wuhan Keri Technology) secondary antibodies, respectively, for $1 \mathrm{~h}$. Immunoreactivity was detected with ECL. Image $J$ software was used to process images.

2.8. Statistical Analysis. Data have been presented as the mean \pm SD. Comparison was conducted with Student's t-test, with the GraphPad Prism (GraphPad Software, La Jolla, California, USA). For statistical analysis, $P<0.05$ was considered as statistically significant.

\section{Results}

3.1. Curcumin Suppresses Proliferation of NPC Cells Positive for $E B V$. To investigate the effects of curcumin treatment on the cellular viability of NPC cells positive for EBV, HONE1, and HK1-EBV, cell proliferation was assessed with the CCK8, after drug administration. As shown in Figure 1, curcumin reduced HONE1 cell viability, in not only a dose-dependent manner but also a time-dependent manner. Moreover, the decline in HONE1 cell viability ranged over 35\%-90\% after treatment with curcumin for $24 \mathrm{~h}$ and $48 \mathrm{~h}$. For the HONE1 cells, the $50 \%$ inhibitory concentration $\left(\mathrm{IC}_{50}\right)$ values were $12.4 \mu \mathrm{M}$ and $3.3 \mu \mathrm{M}$ for the $24 \mathrm{~h}$ and $48 \mathrm{~h}$ curcumin treatment, respectively. On the contrary, similar findings were obtained for the HK1-EBV cells subjected to the treatment series of curcumin. Moreover, after $24 \mathrm{~h}$ and $48 \mathrm{~h}$ curcumin treatment, the HK1-EBV cell viability was reduced from $22 \%$ to $81 \%$. Furthermore, for the HK1-EBV cells, the $\mathrm{IC}_{50}$ values were $10.4 \mu \mathrm{M}$ and $7.2 \mu \mathrm{M}$ for the $24 \mathrm{~h}$ and $48 \mathrm{~h}$ curcumin treatment, respectively (Figure 1(c)). In addition, the concentration series of curcumin treatment slightly inhibited the proliferation of the HUVE and HK2 cells (Figures 1(d) and $1(\mathrm{e})$ ). These findings demonstrate that curcumin could efficiently decrease the $\mathrm{EBV}^{+} \mathrm{NPC}$ cell viabilities but not for the HUVE and HK2 cells.

3.2. Curcumin Leads to Cell Cycle Arrest in NPC Cells Positive for $E B V$. To investigate the effects of curcumin on the cell cycle of NPC cells positive for EBV, flow cytometry was performed after drug treatment. The treatment of $10 \mu \mathrm{M}$ curcumin led to an arrest in the $\mathrm{S}$ phase in the HONE1 cells, with the fraction of S-phased cells increased by $25.6 \%$
(Figure 2). On the contrary, in the HK1-EBV cells, the curcumin treatment $(10 \mu \mathrm{M})$ led to a G2 arrest, with the cell fraction of G2-phase increased by $15.7 \%$. These results reveal that curcumin could induce cell cycle arrest of NPC cells positive for EBV.

\subsection{Curcumin Enhances NPC Cell Apoptosis via Mitochon-} dria- and Death Receptor-Dependent Pathways. Effect of curcumin treatment on NPC cell apoptosis was analyzed. As shown in Figure 3, the curcumin treatment enhanced apoptosis in the HK1-EBV and HONE1 cells, in a dose-dependent manner. Moreover, the apoptosis-related protein expression levels had been examined with the western blotting. Curcumin decreased the caspase- 9 level, while increased the cleaved caspase-3, p53, cleaved PARP-1, Fas, and FasL expression levels, in the HK1-EBV and HONE1 cells (Figure 3 ). The findings suggest that curcumin could induce NPC cell apoptosis via both the mitochondria- and death receptor-dependent pathways.

3.4. Curcumin Downregulated EBNA1 Expression Levels in NPC Cells Positive for EBV. To investigate whether curcumin could alter the expression levels of EBNA1 in the NPC cells positive for EBV after drug administration, the HK1-EBV and HONE1 cells were detected with western blotting. EBNA1 levels in the HONE1 cells were not dramatically altered in the $5 \mu \mathrm{M}$ curcumin treatment group compared with the control group, which were downregulated to $29.8 \%$ for the $10 \mu \mathrm{M}$ curcumin treatment group (Figures 4 (a) and 4(b)). Moreover, the EBNA1 expression levels in the HK1EBV cells were decreased to $30.5 \%$ and $12.7 \%$ of the control group, after curcumin treatment ( 5 and $10 \mu \mathrm{M}$ ). At the same time, another important protein expressed by EBV, LMP1, was also investigated. Our results showed that curcumin could not affect the expression of LMP1 in HONE1 and HK1-EBV cells.

To determine if curcumin specifically decreases the EBNA1 expression levels, HeLa cells were transfected with pSG5-EBNA1 for $4 \mathrm{~h}$, which were then subjected to treatment with or without curcumin. In the pSG5-EBNA1transfected HeLa cells, the EBNA1 level was decreased to $42.5 \%$ and $17.6 \%$ for the treatments of curcumin at $5 \mu \mathrm{M}$ and $10 \mu \mathrm{M}$, respectively (Figure $4(\mathrm{c})$ ). These results were consistent with the results obtained from the cell apoptosis assay.

To further analyze whether the decreased EBNA1 expression level was induced by the alterations at the transcription level, quantitative real-time PCR was carried out for the HK1-EBV and HONE1 cells after drug administration. Our findings showed that compared with the control group, curcumin $(5 \mu \mathrm{M}$ and $10 \mu \mathrm{M})$ reduced the EBNA1 mRNA levels in the HONE1 cells to $70.12 \%$ and $39.52 \%$, respectively. On the contrary, EBNA1 mRNA levels in HK1EBV cells had been reduced to $60.18 \%$ and $37.26 \%$ for the $5 \mu \mathrm{M}$ and $10 \mu \mathrm{M}$ treatment groups, respectively (Figure $4(d))$. The findings suggest that curcumin could inhibit the EBNA1 levels in the HK1-EBV and HONE1 cells. 

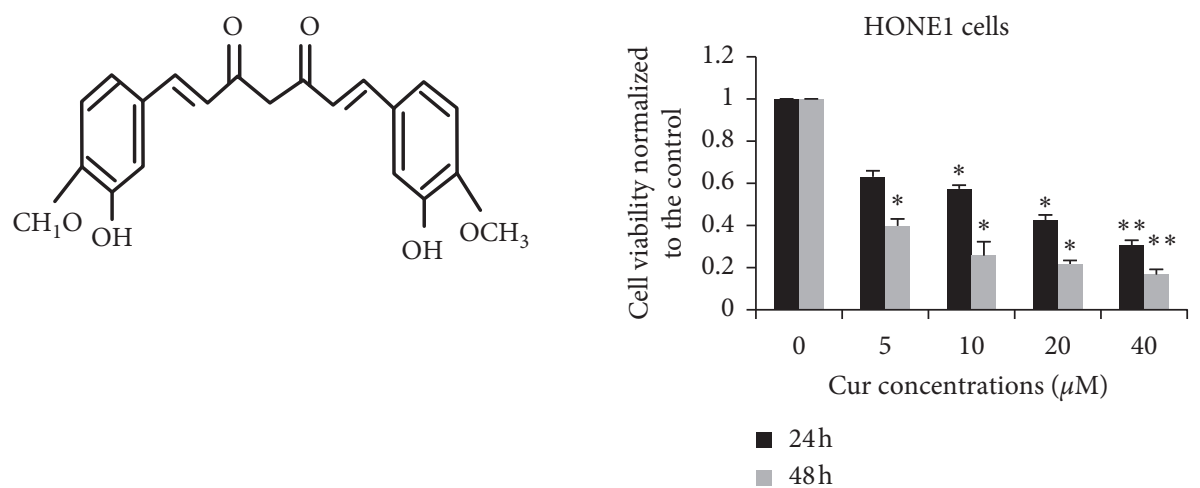

(a)

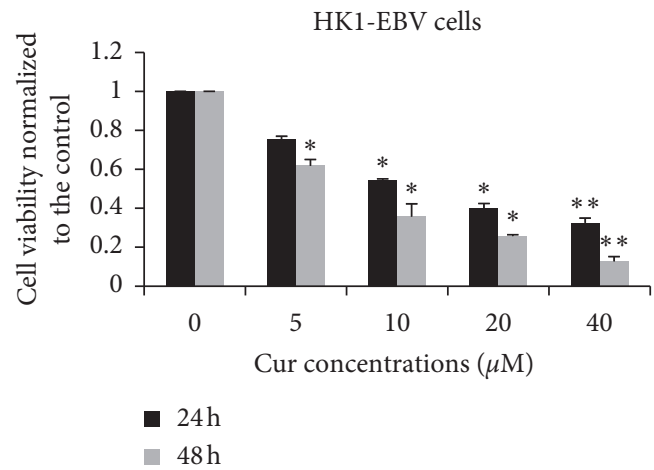

(b)

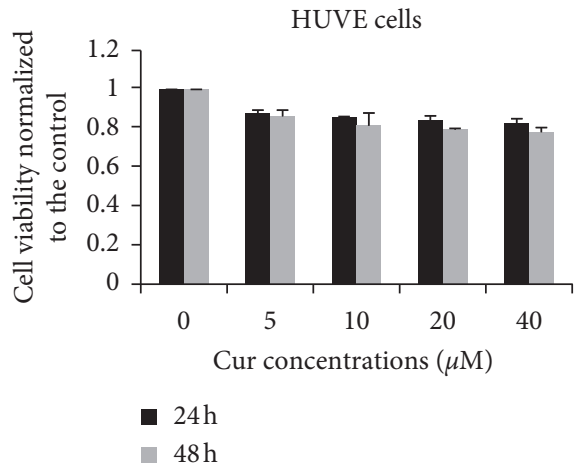

(d)

(c)

HK2 cells

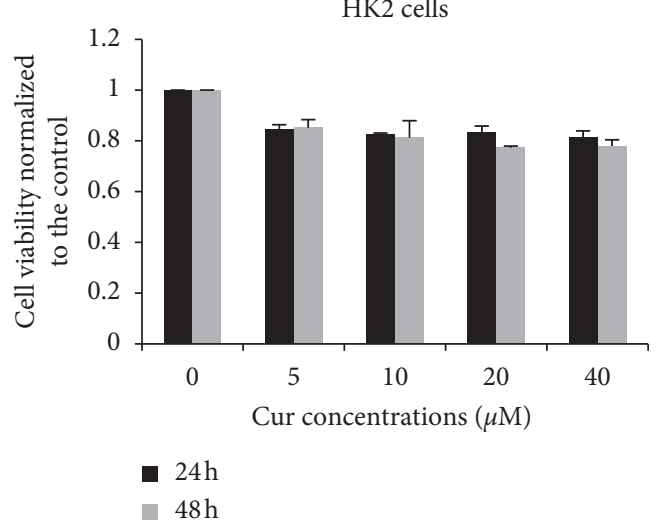

(e)

FIGURE 1: Curcumin suppresses proliferation of EBV-positive NPC cells. (a) Chemical structure of curcumin. HONE1 (b), HK1-EBV (c), HUVE (d), and HK2 (e) cells were treated with DMSO (vehicle control) or curcumin $(5 \mu \mathrm{M}, 10 \mu \mathrm{M}, 20 \mu \mathrm{M}$, or $40 \mu \mathrm{M})$ for $24 \mathrm{~h}$ and $48 \mathrm{~h}$, respectively. Cell proliferation was assessed using the CCK-8. Compared with the vehicle control $\left(0.006 \%\right.$ DMSO) group, ${ }^{*} P<0.05$, ${ }^{* *} P<0.01$.

3.5. Curcumin Decreases EBNA1 Promotor Qp Activities. EBNA1 transcription is initiated by $\mathrm{Qp}$ in $\mathrm{EBV}^{+} \mathrm{NPC}$ cells. To determine the effects of curcumin on the EBNA1 promotor activity, the HeLa cells were transfected with pRL-TK, in combination with pGL3.0 or pGL3.0-Qp vector. After drug administration, the luciferase activity was measured. The Qp activities were reduced to $70.2 \%$ and $59.8 \%$ for the $5 \mu \mathrm{M}$ and $10 \mu \mathrm{M}$ curcumin treatment groups, respectively, (Figure 4(e)). Thus, curcumin treatment could decrease the transcriptional activity of EBNA1 in the HeLa cells.
3.6. Curcumin Reduces Stability and Promotes Proteasomal Degradation of EBNA1. To determine the effects of curcumin on the stability of EBNA1, the HONE1 and HK1-EBV cells were subjected to curcumin, under the condition of cycloheximide (CHX). Curcumin reduced the half-time of EBNA1 in HK1-EBV and HONE1 cells after CHX treatment compared with the control groups (Figures $4(\mathrm{f})$ and $4(\mathrm{~g})$ ).

In addition, the effects of curcumin on the proteasome pathway were investigated. HK1-EBV and HONE1 cells were incubated with curcumin, with or without the proteasome inhibitor (MG-132). MG-132 treatment 


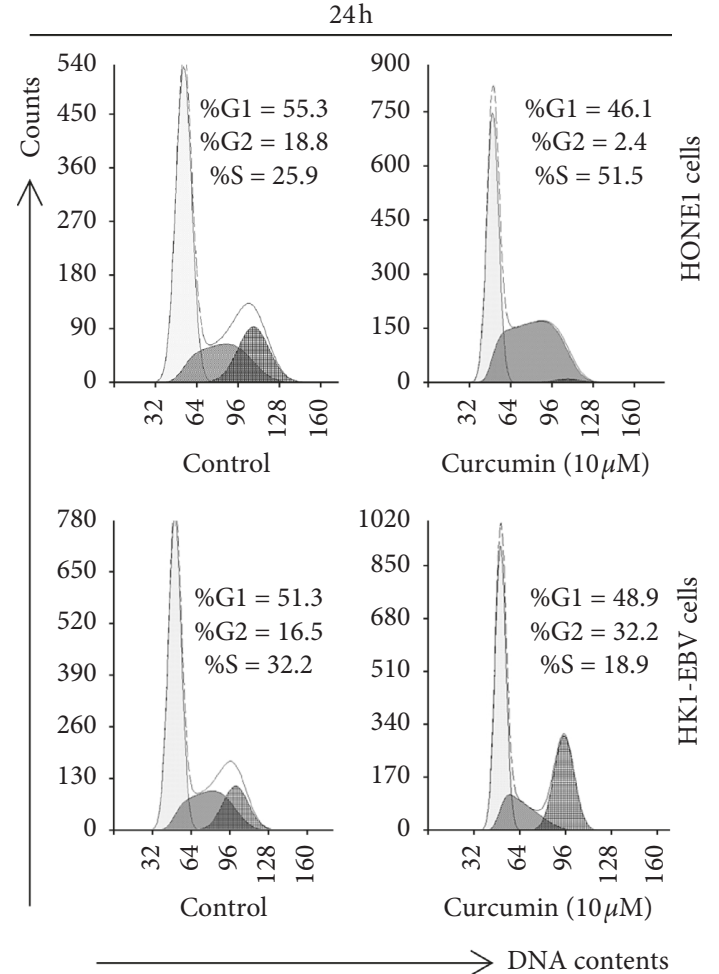

(a)

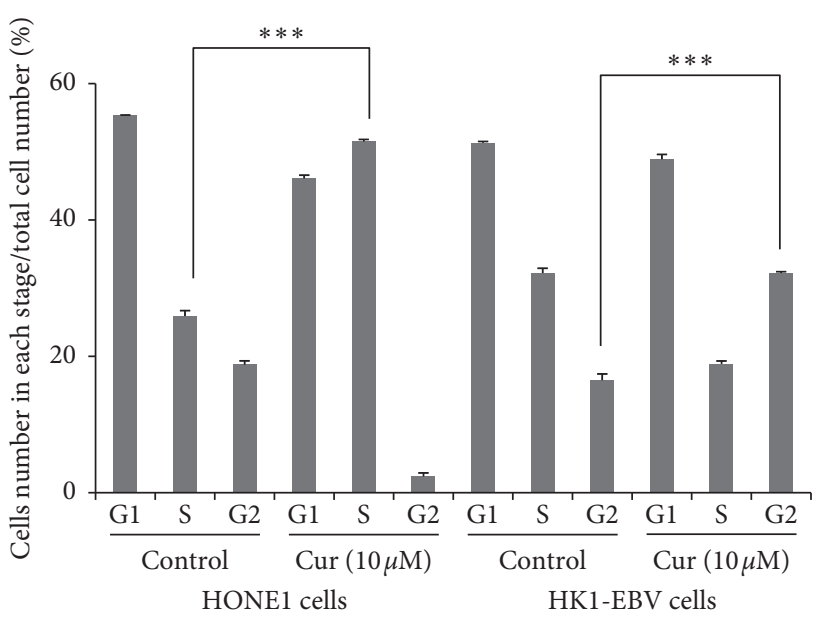

(b)

FIgUre 2: Curcumin induces cell cycle arrest in EBV-positive NPC cells. (a) Effect of curcumin on the cell cycle. HONE1 and HK1-EBV cells were treated with DMSO (vehicle control) or curcumin at indicated concentrations, for $24 \mathrm{~h}$. Cell cycle was analyzed by flow cytometry. (b) Statistical analysis of the cell cycle. Compared with the vehicle control $\left(0.006 \%\right.$ DMSO) group, ${ }^{*} P<0.05,{ }^{* *} P<0.01$.

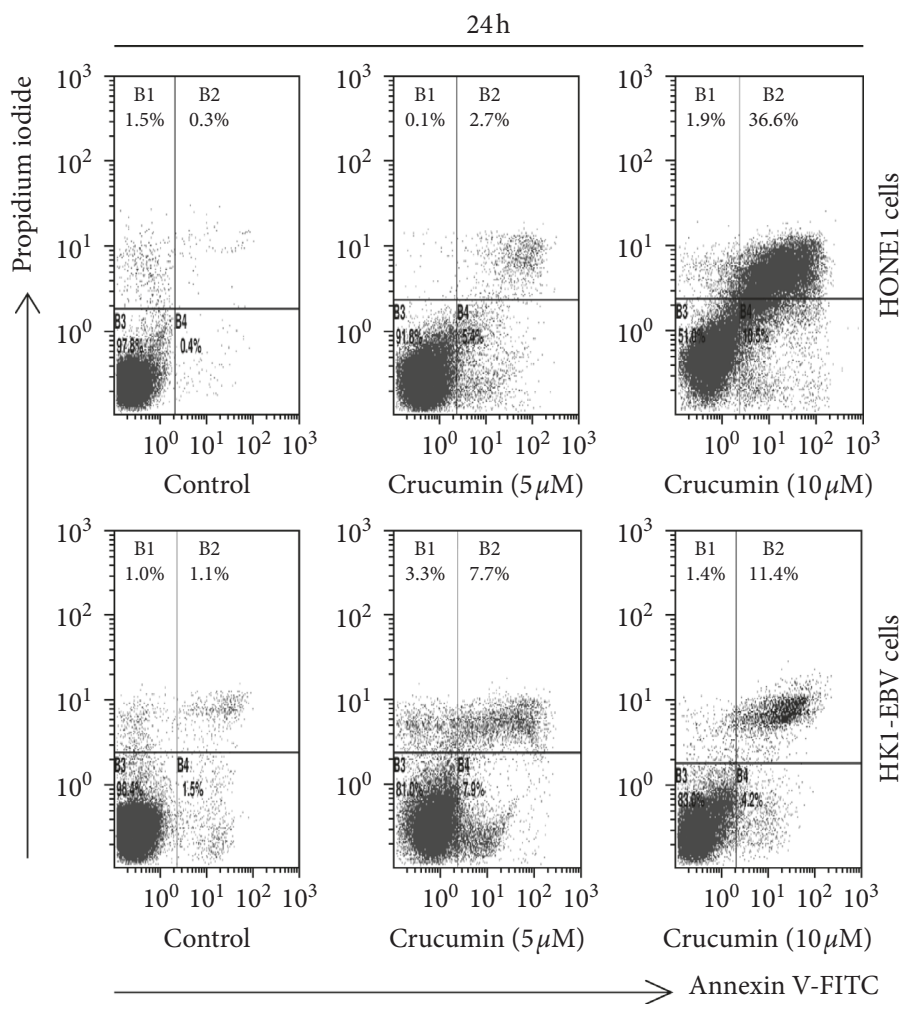

(a)

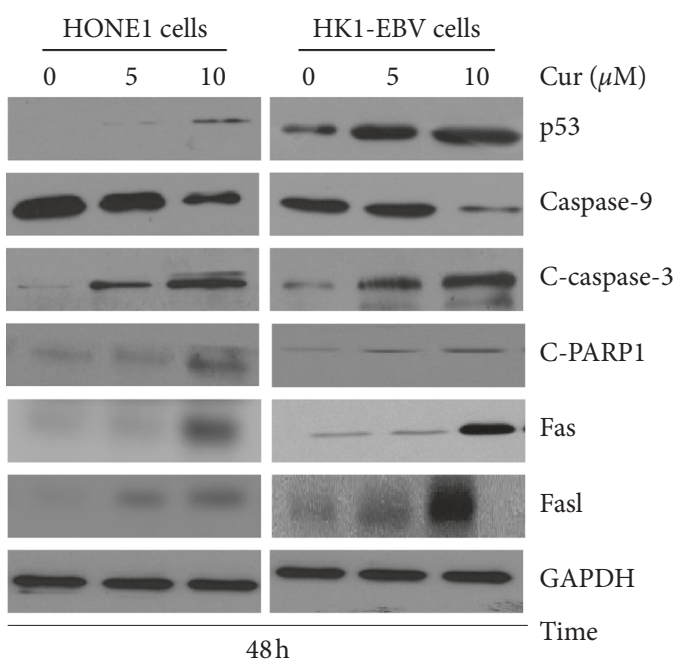

(b)

FIgURE 3: Curcumin induces cell apoptosis in EBV-positive NPC cells. (a) Effect of curcumin on cell apoptosis. HONE1 and HK1-EBV cells were treated with DMSO (vehicle control) or curcumin at indicated concentrations, for $24 \mathrm{~h}$. Cellular apoptosis was analyzed by flow cytometry. (b) HONE1 and HK1-EBV cells were treated with DMSO (vehicle control) or curcumin at indicated concentrations, for $48 \mathrm{~h}$, and the related protein expression levels were determined by western blot analysis. 


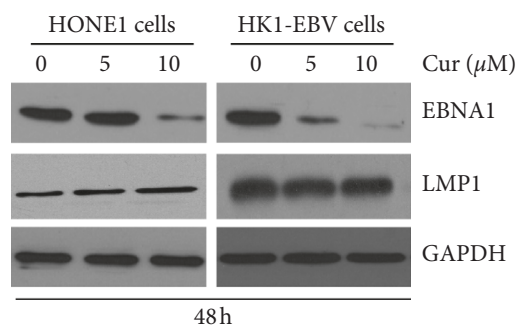

(a)

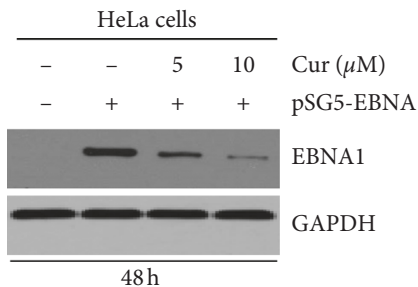

(c)

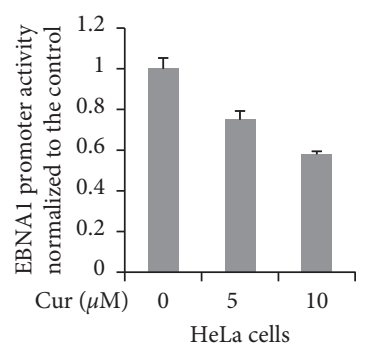

(e)

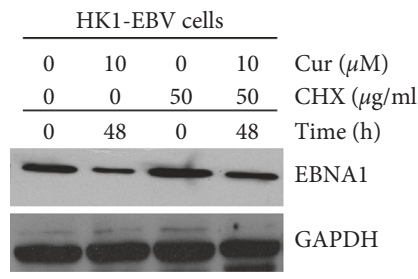

(g)

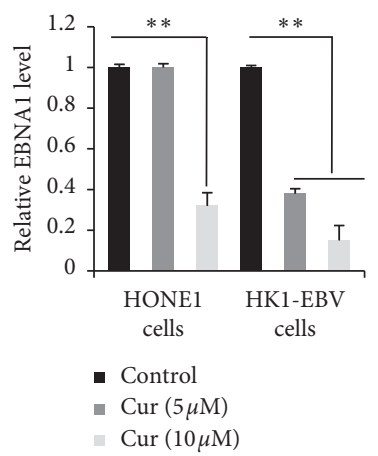

(b)

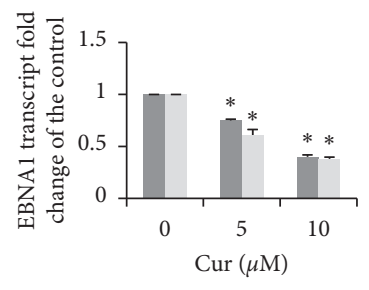

- HONE1

- HK1-EBV

(d)

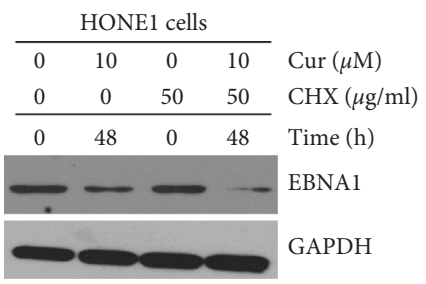

(f)

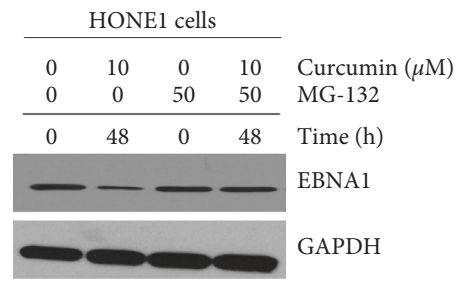

(h)

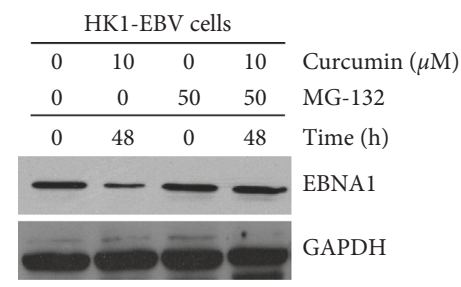

(i)

FIGURE 4: Effects of curcumin on transcription, expression, and half-time of EBNA1 in HONE1 and HK1-EBV cells. (a) HONE1 and HK1-EBV cells treated with DMSO (vehicle control) or curcumin $(5 \mu \mathrm{M}$ and $10 \mu \mathrm{M})$, for $48 \mathrm{~h}$. (b) Statistical analysis of the expression levels of EBNA1 in the HONE1 and HK1-EBV cells. (c) HeLa cells transfected with pSG5 or pSG5-EBNA1, followed by the treatment of curcumin $(5 \mu \mathrm{M}$ and $10 \mu \mathrm{M})$ for $44 \mathrm{~h}$ (d) HONE1 and HK1-EBV cells treated with DMSO (vehicle control) or curcumin ( $5 \mu \mathrm{M}$ and $10 \mu \mathrm{M}$ ), for $24 \mathrm{~h}$ (e) HeLa cells cotransfected with pRL-TK and pGL3.0-Qp, followed by the treatment with DMSO (vehicle control) or curcumin $(5 \mu \mathrm{M}$ and $10 \mu \mathrm{M})$. The activity of the promoter was determined by the Dual-Luciferase reporter assay. The HONE1 (f) and HK1-EBV (g) cells treated with DMSO (vehicle control) or curcumin $(10 \mu \mathrm{M})$, in the presence or absence of CHX $(50 \mu \mathrm{g} / \mathrm{ml})$. The HONE1 (h) and HK1-EBV (i) cells treated with DMSO (vehicle control) or curcumin $(10 \mu \mathrm{M})$, in the presence or absence of MG-132 $(50 \mu \mathrm{g} / \mathrm{ml})$. Transcription levels of EBNA1 were determined by quantitative real-time PCR. Western blot analysis was performed to detect the protein expression levels. Compared with the vehicle control $(0.006 \% \mathrm{DMSO})$ group, ${ }^{*} P<0.05,{ }^{* *} P<0.01$. 
increased the EBNA1 expression levels (Figures 4(h) and 4(i)) in both the HK1-EBV and HONE1 cells, either with or without curcumin. These findings suggest that curcumin could induce the EBNA1 degradation via the proteasomeubiquitin pathway.

3.7. EBNA1 Overexpression Attenuates Effects of Curcumin. Whether the curcumin-decreased cell viability was induced by the inhibited EBNA1 expression was determined. The HK1 and HONE1 cells were transfected with pSG5-EBNA1 and then treated with curcumin. The transient transfection increased the EBNA1 expression levels in the HONE1 cells by $301.3 \%$ and in the HK1 cells by $368.1 \%$ (Figures 5 (a) and $5(\mathrm{~b})$ ).

The cellular viability was then determined. As shown in Figures 5(c) and 5(d), curcumin treatment significantly decreased the cell viabilities of both HK1-EBV and HONE1 cells. After EBNA1 overexpression for $48 \mathrm{~h}$, the cell viabilities of HONE1 and HK1-EBV cells were increased by $15.8 \%$ and $10.4 \%$, respectively. The findings demonstrate that curcumin could inhibit $\mathrm{EBV}^{+} \mathrm{HONE} 1$ and HK1-EBV cell proliferation, probably via decreasing EBNA1 expression.

3.8. Curcumin Inhibits Replication of EBV in NPC Cells Positive for EBV. Effect of curcumin on the replication of EBV was determined. The TPA and NaB-induced HK1EBV and HONE1 cells were treated with curcumin. Quantitative real-time PCR was performed to investigate the latent and lytic replication of EBV EBNA1 fragment. Curcumin treatment reduced the production of progeny virion, in a dose-dependent manner (Figures 6(a) and 6(b)). The findings demonstrate that curcumin could inhibit EBV latent and lytic replication in both HK1-EBV and HONE1 cells. Moreover, the effect of Curcumin on the EBV transcriptional activator BZLF1 encoded by IE was investigated with western blot analysis. Our results showed that curcumin significantly reduced the expression levels of BZLF1 in both induced and noninduced HONE1 and HK1-EBV cells.

\section{Discussion}

It has been demonstrated that the infection of EBV is closely linked to NPC [28]. EBNA1 could regulate the DNA synthesis of EBV, which contributes to the cellular proliferation, survival, tumorigenesis, and immortalization, which provides an attractive target for the EBV-related diseases $[4,29,30]$. Previous studies from our lab have shown that the Hsp90 inhibitor and berberine could inhibit the proliferation of malignant cells infected with EBV via the EBNA1-dependent pathway $[13,25]$. It is clinically significant to find and develop the drugs that could inhibit the activity of EBNA1. Herein, our findings indicated that curcumin reduced the EBNA1 expression via the proteasome-ubiquitin pathway, both in vivo and in vitro. Moreover, EBNA1 overexpression could attenuate the curcumin-induced NPC cell apoptosis.
Curcumin is a rhizome extracted from the herb Curcuma longa Linn., which is frequently used in Ayurveda and traditional Chinese medicine to prevent and cure a variety of ailments in human beings [31, 32]. It has been shown that curcumin might be applied as efficient treatments for various disorders, including the allergy, coryza, sinusitis, hepatic diseases, cough, asthma, and bronchial hyperactivity [33-35]. Moreover, curcumin could exert the antioxidant, antiarthritic, hepatoprotective, thrombosuppressive, and cardioprotective effects [36, 37]. Furthermore, due to its distinct chemical properties, curcumin has also been reported as a potent anticancer compound $[38,39]$. Curcumin could interact with various intracellular and extracellular molecules actively implied in the initiation as well as progression of cancers, thereby inhibiting the disease progression [40].

Increasing evidence has suggested that the inflammatory pathways play pivotal roles in a variety of chronic diseases, including cancers [41]. EBNA1 is a vital protein for the replication and persistence of EBV episomes $[12,42]$. Herein, curcumin reduced the EBNA1 level in the $\mathrm{EBV}^{+} \mathrm{NPC}$ cells and $\mathrm{EBV}^{-} \mathrm{HeLa}$ cells, which had pSG5-EBNA1 transfection. In addition, the EBNA1 stability was declined by curcumin treatment. Curcumin could inhibit the mRNA and protein levels of EBNA1 in the HK1 and HONE1 cells.

A previous study from our lab has indicated that triptolide could suppress the LMP1 promoter ED-L1 activity in the B lymphocytes positive for latency III type infected EBV [26]. In NPC, the EBV would expresse type II latency. Herein, the EBNA1 mRNA expression levels were decreased by curcumin treatment. Moreover, the EBNA1 promoter Qp activity was inhibited by the curcumin treatment, indicating that the decreased activity of the EBNA1 promoter would inhibit the transcription levels of EBNA1.

Previous studies have suggested that curcumin could activate caspase- 3 , caspase- 8 , and caspase- 9 and PARP protein in various cancer cell lines $[43,44]$. However, increasing evidence has demonstrated that the essential apoptotic pathways represent the mitochondria-dependent and death receptor-mediated pathways [45]. In this study, our results suggested that curcumin could induce $\mathrm{EBV}^{+}$ NPC cell apoptosis, through the caspase-9-involved and death receptor-mediated pathways.

In addition to the EBV-encoded protein latent membrane protein 1 (LMP1), EBNA1 also regulates the viral transcription and exerts multiple functions on cellular proteins, as well as on the pathways promoting cell survival and proliferation [14]. A previous study from our lab has shown that the LMP1 overexpression would increase the $\mathrm{EBV}^{+}$B lymphocyte (B95-8 and P3HR) viabilities [26]. Therefore, we speculate whether the EBNA1 overexpression could increase the viabilities of HK1-EBV and HONE1 cells. Our findings showed that EBNA1 overexpression attenuated the inhibitory effects of curcumin. Moreover, the curcumin treatment reduced the $\mathrm{EBV}^{+}$NPC cell proliferation and activities, at least partially, related to the reduced EBNA1 level. 


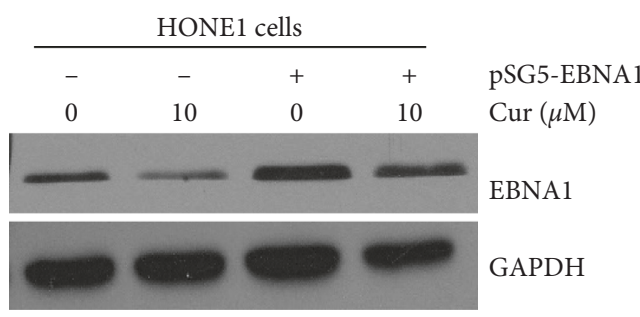

(a)

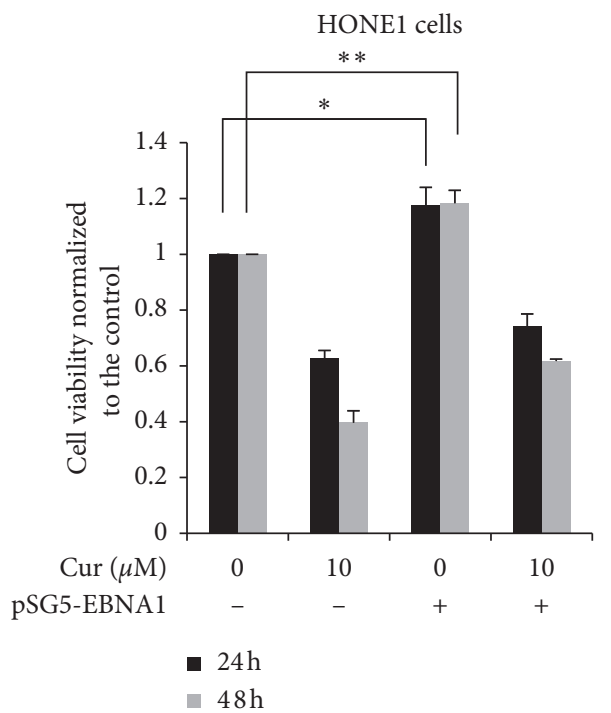

(c)

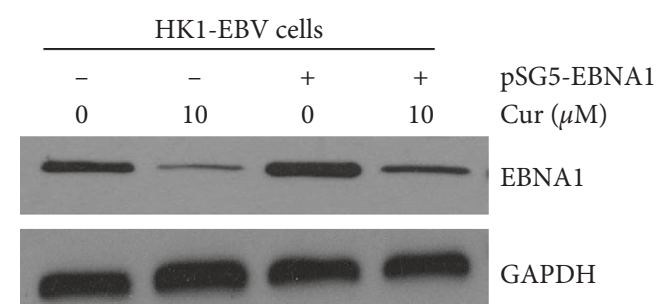

(b)

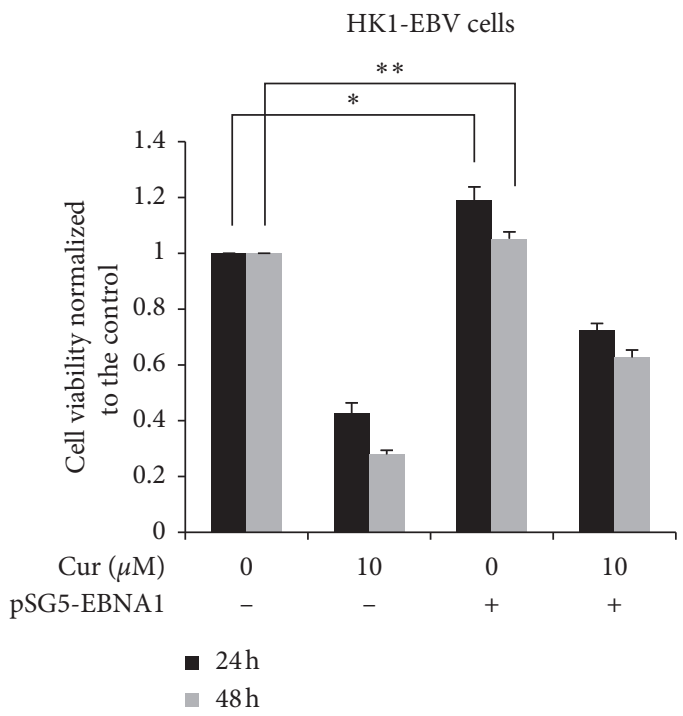

(d)

FIGURE 5: EBNA1 overexpression attenuates curcumin-induced cell killing and apoptosis-promoting effects in EBV-positive NPC cells. The HONE1 (a) and HK1-EBV (b) cells were transiently transfected with empty vector or pSG5-EBNA1 for $4 \mathrm{~h}$ followed by the treatment of curcumin $(10 \mu \mathrm{M})$. Western blot analysis was performed to detect the protein expression levels. Cell viabilities of HONE1 (c) and HK1-EBV (d) cells were determined by the CCK- 8 . Compared with the vehicle control (0.006\% DMSO) group, ${ }^{*} P<0.05,{ }^{* *} P<0.01$.

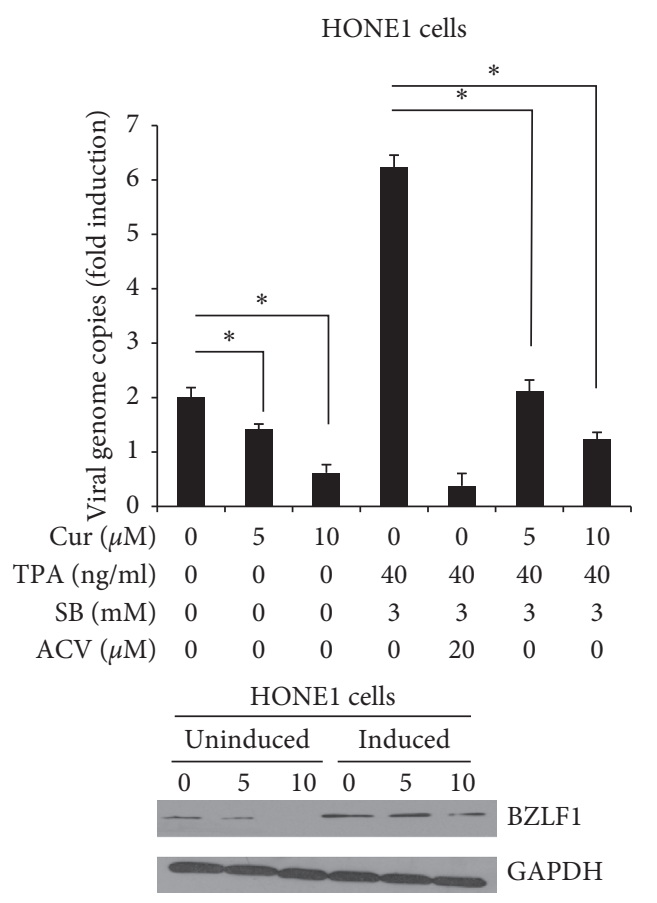

(a)
HK1-EBV cells

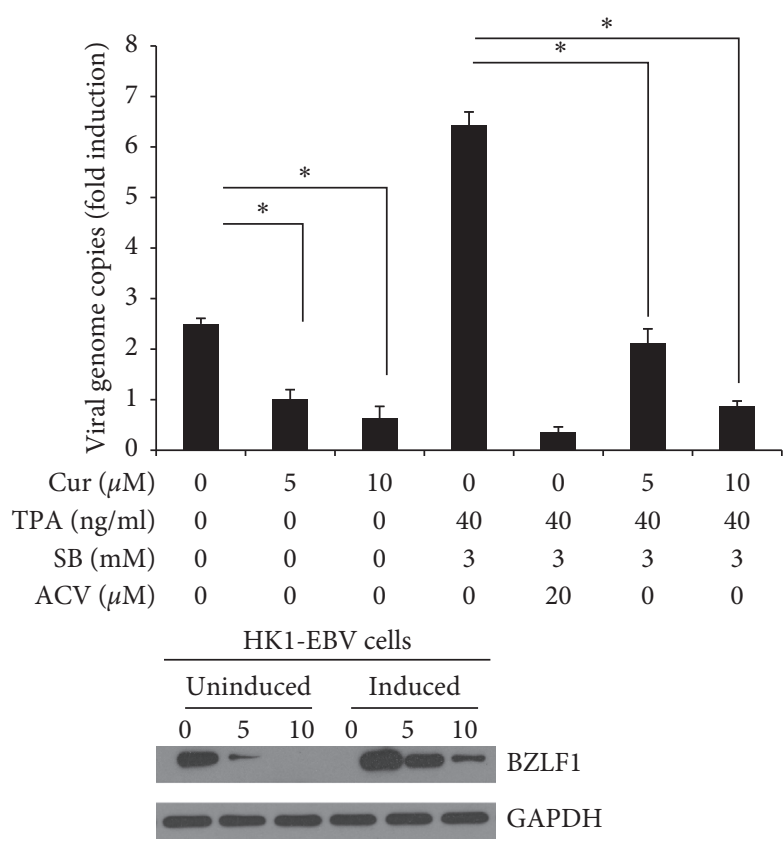

(b)

FIgURE 6: Curcumin reduces EBV virion production in EBV-positive NPC cells. The HONE1 (a) and HK1-EBV (b) cells were treated with TPA $(40 \mathrm{ng} / \mathrm{ml})$ and SB $(3 \mathrm{mM})$ for $3 \mathrm{~h}$ followed by the treatment of DMSO (vehicle control) or curcumin $(5 \mu \mathrm{M}$ and $10 \mu \mathrm{M})$. The culture medium was collected and subjected to quantitative real-time PCR. Compared with the vehicle control (0.006\% DMSO) group, ${ }^{*} P<0.05,{ }^{* *} P<0.01$. 


\section{Conclusions}

In conclusion, our findings indicate that curcumin reduced cell proliferation and apoptosis and changed cycle arrest in the $\mathrm{EBV}^{+}$NPC cells. Moreover, the decreased Qp promoter activity provides evidence for curcumin-induced declined EBNA1 mRNA levels. In addition, EBNA1 overexpression attenuates the effects of curcumin in $\mathrm{EBV}^{+} \mathrm{NPC}$ cells. Based on these findings, curcumin might make a novel therapy for a variety of tumors in clinics, including NPC. Finally, although our results suggested that curcumin might reduce the total number of EBV-positive cells in vitro, the in vivo test should be carried out, and the long-term toxicity of curcumin should be estimated. Moreover, the safety and efficacy of curcumin would need to be investigated in clinical trials.

\section{Data Availability}

The data used to support the findings of this study are available from the corresponding author upon request.

\section{Conflicts of Interest}

The authors declare that there are no conflicts of interest regarding the publication of this paper.

\section{Acknowledgments}

This work was supported by the Science and Technology Bureau of Weifang Kuiwen District (No. 2018-3).

\section{References}

[1] L. Fitzsimmons and G. L. Kelly, "EBV and apoptosis: the viral master regulator of cell fate?," Viruses, vol. 9, no. 11, 2017.

[2] Y.-J. Xu, R. Zhou, J.-F. Zong et al., "Epstein-Barr virus-coded miR-BART13 promotes nasopharyngeal carcinoma cell growth and metastasis via targeting of the NKIRAS2/NF- $\kappa \mathrm{B}$ pathway," Cancer Letters, vol. 447, pp. 33-40, 2019.

[3] D. Elgui de Oliveira, B. G. Müller-Coan, and J. S. Pagano, "Viral carcinogenesis beyond malignant transformation: EBV in the progression of human cancers," Trends in Microbiology, vol. 24, no. 8, pp. 649-664, 2016.

[4] J. Dheekollu, K. Malecka, A. Wiedmer et al., "Carcinoma-risk variant of EBNA1 deregulates Epstein-Barr virus episomal latency," Oncotarget, vol. 8, no. 5, pp. 7248-7264, 2017.

[5] Y. Nakanishi, N. Wakisaka, S. Kondo et al., "Progression of understanding for the role of Epstein-Barr virus and management of nasopharyngeal carcinoma," Cancer and Metastasis Reviews, vol. 36, no. 3, pp. 435-447, 2017.

[6] J. Zhang, L. Jia, C. M. Tsang, and S. W. Tsao, "EBV infection and glucose metabolism in nasopharyngeal carcinoma," Advances in Experimental Medicine and Biology, vol. 1018, pp. 75-90, 2017.

[7] A. Nanbo, H. Katano, M. Kataoka et al., "Infection of Epstein-Barr virus in type III latency modulates biogenesis of exosomes and the expression profile of exosomal miRNAs in the Burkitt lymphoma mutu cell lines," Cancers, vol. 10, no. 7, p. 237, 2018.

[8] L. Jiang, C. Xie, H. L. Lung et al., "EBNA1-targeted inhibitors: novel approaches for the treatment of Epstein-Barr virus- associated cancers," Theranostics, vol. 8, no. 19, pp. 53075319, 2018.

[9] J. S. Deakyne, K. A. Malecka, T. E. Messick, and P. M. Lieberman, "Structural and functional basis for an EBNA1 hexameric ring in Epstein-Barr virus episome maintenance," Journal of Virology, vol. 91, no. 19, 2017.

[10] M. Albanese, T. Tagawa, M. Bouvet et al., "Epstein-Barr virus microRNAs reduce immune surveillance by virus-specific CD $8^{+}$T cells," Proceedings of the National Academy of Sciences, vol. 113, no. 42, pp. E6467-E6475, 2016.

[11] S. AlQarni, Y. Al-Sheikh, D. Campbell et al., "Lymphomas driven by Epstein-Barr virus nuclear antigen-1 (EBNA1) are dependant upon Mdm2," Oncogene, vol. 37, no. 29, pp. 3998-4012, 2018.

[12] R. J. A. Verhoeven, S. Tong, J. Zong et al., "NF-kappaB signaling regulates epstein-barr virus BamHI-Q-driven EBNA1 expression," Cancers, vol. 10, no. 4, p. 119, 2018.

[13] C. Wang, H. Wang, Y. Zhang et al., "Berberine inhibits the proliferation of human nasopharyngeal carcinoma cells via an Epstein-Barr virus nuclear antigen 1-dependent mechanism," Oncology Reports, vol. 37, no. 4, pp. 2109-2120, 2017.

[14] Y. Zhang, H. Wang, Y. Liu et al., "Baicalein inhibits growth of Epstein-Barr virus-positive nasopharyngeal carcinoma by repressing the activity of EBNA1 Q-promoter," Biomedicine \& Pharmacotherapy, vol. 102, pp. 1003-1014, 2018.

[15] S. Mapoung, S. Suzuki, S. Fuji et al., "Cyclohexanone curcumin analogs inhibit the progression of castration-resistant prostate cancer in vitro and in vivo," Cancer Science, vol. 110, no. 2, pp. 596-607, 2019.

[16] T. Tsuda, "Curcumin as a functional food-derived factor: degradation products, metabolites, bioactivity, and future perspectives," Food \& Function, vol. 9, no. 2, pp. 705-714, 2018.

[17] K.-Y. Chin, "The spice for joint inflammation: anti-inflammatory role of curcumin in treating osteoarthritis," Drug Design, Development and Therapy, vol. 10, pp. 3029-3042, 2016.

[18] P. V. Sanitá, A. C. Pavarina, L. N. Dovigo, A. P. D. Ribeiro, M. C. Andrade, and E. G. D. O. Mima, "Curcumin-mediated anti-microbial photodynamic therapy against Candida dubliniensis biofilms," Lasers in Medical Science, vol. 33, no. 4, pp. 709-717, 2018

[19] Y. Yao, W. Wang, M. Li et al., "Curcumin exerts its antihypertensive effect by down-regulating the AT1 receptor in vascular smooth muscle cells," Scientific Reports, vol. 6, no. 1, p. 25579, 2016.

[20] J. Adiwidjaja, A. J. McLachlan, and A. V. Boddy, "Curcumin as a clinically-promising anti-cancer agent: pharmacokinetics and drug interactions," Expert Opinion on Drug Metabolism \& Toxicology, vol. 13, no. 9, pp. 953-972, 2017.

[21] K. Yoshida, S. Toden, P. Ravindranathan, H. Han, and A. Goel, "Curcumin sensitizes pancreatic cancer cells to gemcitabine by attenuating PRC2 subunit EZH2, and the lncRNA PVT1 expression," Carcinogenesis, vol. 38, no. 10, pp. 1036-1046, 2017.

[22] S. Hu, Y. Xu, L. Meng, L. Huang, and H. Sun, "Curcumin inhibits proliferation and promotes apoptosis of breast cancer cells," Experimental and Therapeutic Medicine, vol. 16, no. 2, pp. 1266-1272, 2018.

[23] A. A. Momtazi-Borojeni, F. Ghasemi, A. Hesari, M. Majeed, M. Caraglia, and A. Sahebkar, "Anti-cancer and radio-sensitizing effects of curcumin in nasopharyngeal carcinoma," Current Pharmaceutical Design, vol. 24, no. 19, pp. 2121-2128, 2018. 
[24] F. W. Yang, J. Z. Huang, X. L. Lin, Z. N. Zhen, and X. M. Chen, "Apoptosis in nasopharyngeal carcinoma cell line NCE induced by curcumin and its molecular mechanism," Zhonghua Er Bi Yan Hou Tou Jing Wai Ke Za Zhi, vol. 41, no. 8, pp. 612-616, 2006.

[25] X. Sun, E. A. Barlow, S. Ma et al., "Hsp90 inhibitors block outgrowth of EBV-infected malignant cells in vitro and in vivo through an EBNA1-dependent mechanism," Proceedings of the National Academy of Sciences, vol. 107, no. 7, pp. 3146-3151, 2010

[26] H. Zhou, W. Guo, C. Long, H. Wang, J. Wang, and X. Sun, "Triptolide inhibits proliferation of Epstein-Barr virus-positive B lymphocytes by down-regulating expression of a viral protein LMP1," Biochemical and Biophysical Research Communications, vol. 456, no. 3, pp. 815-820, 2015.

[27] C. Long, J. Wang, W. Guo et al., "Triptolide inhibits transcription of hTERT through down-regulation of transcription factor specificity protein 1 in primary effusion lymphoma cells," Biochemical and Biophysical Research Communications, vol. 469, no. 1, pp. 87-93, 2016.

[28] S. W. Tsao, C. M. Tsang, and K. W. Lo, "Epstein-Barr virus infection and nasopharyngeal carcinoma," Philosophical Transactions of the Royal Society B: Biological Sciences, vol. 372, no. 1732, 2017.

[29] L. Frappier, "Contributions of Epstein-Barr nuclear antigen 1 (EBNA1) to cell immortalization and survival," Viruses, vol. 4, no. 9, pp. 1537-1547, 2012.

[30] I. Tempera, A. De Leo, A. V. Kossenkov et al., "Identification of MEF2B, EBF1, and IL6R as direct gene targets of epsteinbarr virus (EBV) nuclear antigen 1 critical for EBV-infected B-lymphocyte survival," Journal of Virology, vol. 90, no. 1, pp. $345-355,2016$.

[31] T. Arablou and R. Kolahdouz-Mohammadi, "Curcumin and endometriosis: review on potential roles and molecular mechanisms," Biomedicine \& Pharmacotherapy, vol. 97, pp. 91-97, 2018.

[32] P. P. Desai and V. B. Patravale, "Curcumin cocrystal micellesmultifunctional nanocomposites for management of neurodegenerative ailments," Journal of Pharmaceutical Sciences, vol. 107, no. 4, pp. 1143-1156, 2018.

[33] L. Birdane, C. Cingi, N. B. Muluk, T. San, and D. Burukoglu, "Evaluation of the efficacy of curcumin in experimentally induced acute sinusitis in rats," Ear, Nose \& Throat Journal, vol. 95, no. 12, pp. E21-E27, 2016.

[34] J. Liang, X. Dong, A. Yang, D. Zhu, D. Kong, and F. Lv, “A dual fluorescent reverse targeting drug delivery system based on curcumin-loaded ovalbumin nanoparticles for allergy treatment," Nanomedicine: Nanotechnology, Biology and Medicine, vol. 16, pp. 56-68, 2019.

[35] S. Saadati, B. Hatami, Z. Yari et al., "The effects of curcumin supplementation on liver enzymes, lipid profile, glucose homeostasis, and hepatic steatosis and fibrosis in patients with non-alcoholic fatty liver disease," European Journal of Clinical Nutrition, vol. 73, no. 3, pp. 441-449, 2019.

[36] H. Avci, E. T. Epikmen, E. Ipek et al., "Protective effects of silymarin and curcumin on cyclophosphamide-induced cardiotoxicity," Experimental and Toxicologic Pathology, vol. 69, no. 5, pp. 317-327, 2017.

[37] C. Chang, T. G. Meikle, Y. Su et al., "Encapsulation in egg white protein nanoparticles protects anti-oxidant activity of curcumin," Food Chemistry, vol. 280, pp. 65-72, 2019.

[38] T. Feng, Y. Wei, R. Lee, and L. Zhao, "Liposomal curcumin and its application in cancer," International Journal of Nanomedicine, vol. 12, pp. 6027-6044, 2017.
[39] M. Namdari and A. Eatemadi, "Cardioprotective effects of curcumin-loaded magnetic hydrogel nanocomposite (nanocurcumin) against doxorubicin-induced cardiac toxicity in rat cardiomyocyte cell lines," Artificial Cells, Nanomedicine, and Biotechnology, vol. 45, no. 4, pp. 731-739, 2017.

[40] M. I. Qadir, S. T. Naqvi, and S. A. Muhammad, "Curcumin: a polyphenol with molecular targets for cancer control," Asian Pacific Journal of Cancer Prevention, vol. 17, no. 6, pp. 2735-2739, 2016.

[41] L. Huan, L. H. Liang, and X. H. He, "Role of microRNAs in inflammation-associated liver cancer," Cancer Biology \& Medicine, vol. 13, no. 4, pp. 407-425, 2016.

[42] K.-W. Noh, J. Park, and M.-S. Kang, "Targeted disruption of EBNA1 in EBV-infected cells attenuated cell growth," BMB Reports, vol. 49, no. 4, pp. 226-231, 2016.

[43] N. S. Srivastava and R. A. K. Srivastava, "Curcumin and quercetin synergistically inhibit cancer cell proliferation in multiple cancer cells and modulate $\mathrm{Wnt} / \beta$-catenin signaling and apoptotic pathways in A375 cells," Phytomedicine, vol. 52, pp. 117-128, 2019.

[44] Z. Zhao, C. Li, H. Xi, Y. Gao, and D. Xu, "Curcumin induces apoptosis in pancreatic cancer cells through the induction of forkhead box O1 and inhibition of the PI3K/Akt pathway," Molecular Medicine Reports, vol. 12, no. 4, pp. 5415-5422, 2015.

[45] H. Hu, M. Tian, C. Ding, and S. Yu, "The C/EBP homologous protein (CHOP) transcription factor functions in endoplasmic reticulum stress-induced apoptosis and microbial infection," Frontiers in Immunology, vol. 9, p. 3083, 2019. 


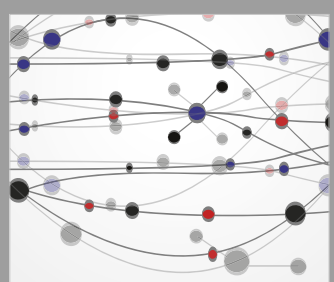

The Scientific World Journal
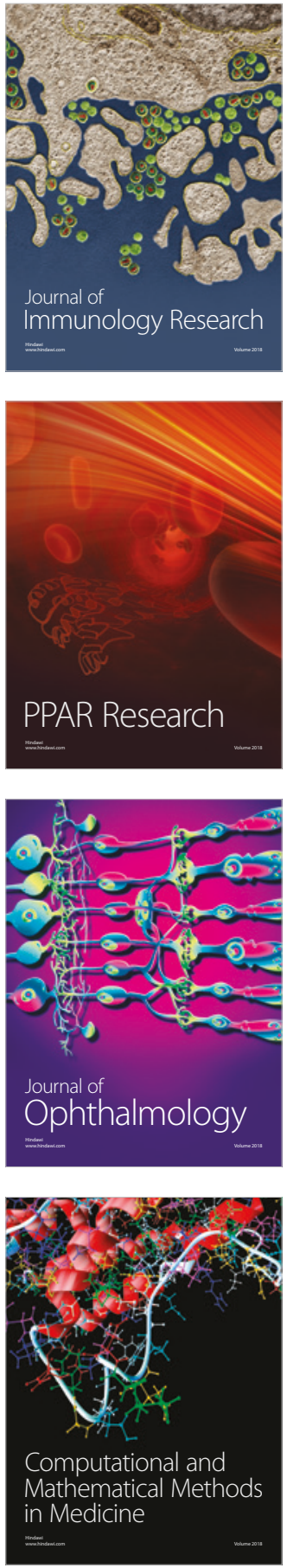

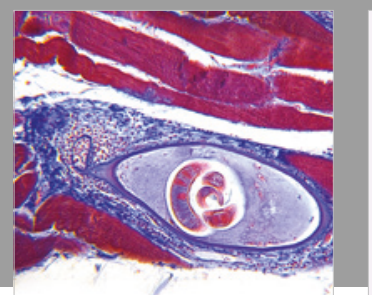

Gastroenterology Research and Practice

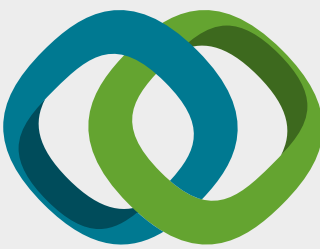

\section{Hindawi}

Submit your manuscripts at

www.hindawi.com
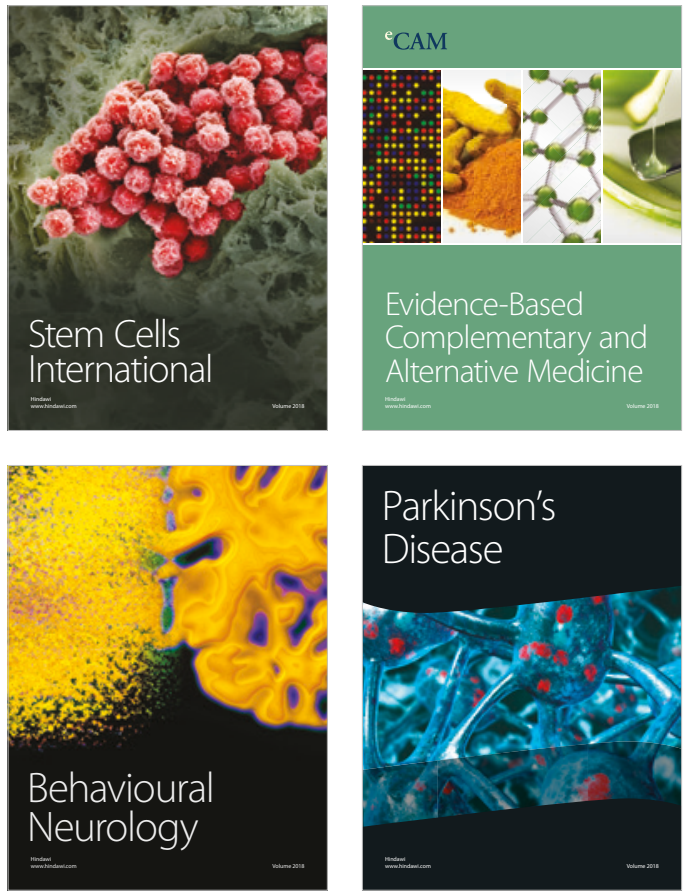

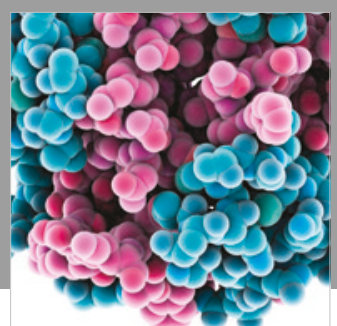

ournal of

Diabetes Research

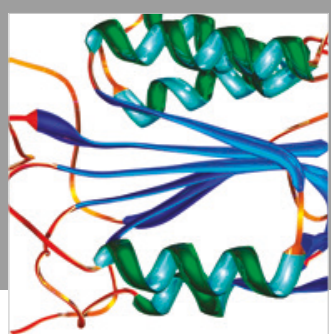

Disease Markers
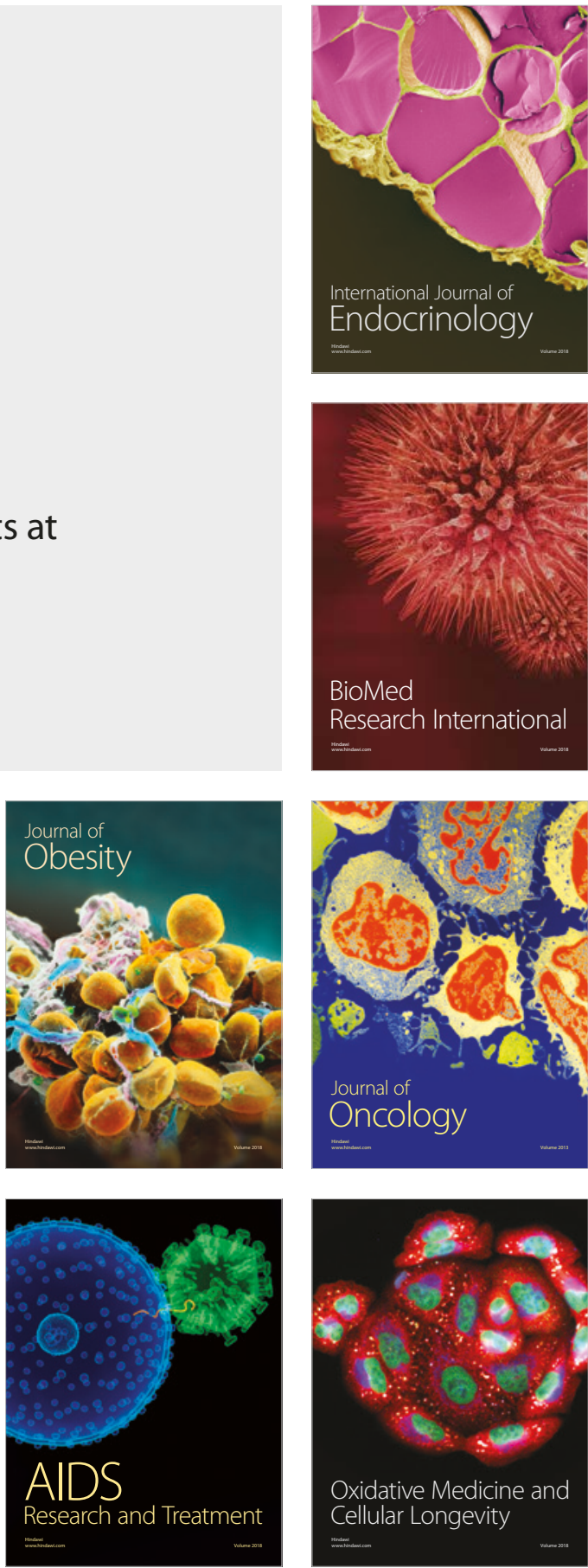